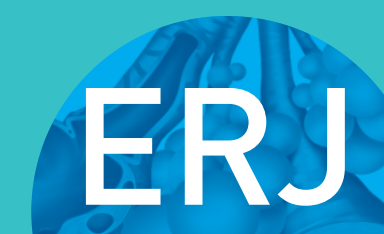

open research
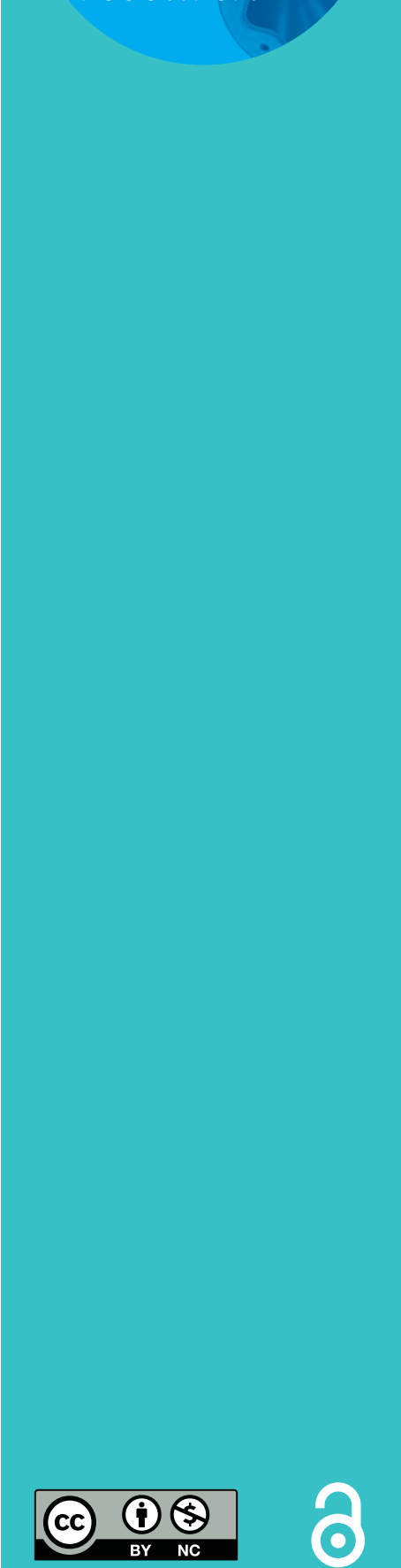

\section{Understanding the priorities for women diagnosed with lymphangioleiomyomatosis: a patient perspective}

\author{
Iris Bassi ${ }^{1}$, Gill Hollis ${ }^{2}$, Vincent Cottin ${ }^{3}$, Sergio Harari ${ }^{4}$, Elma Zwanenburg ${ }^{5}$, \\ Marcel Veltkamp ${ }^{5}$, Alvaro Casanova ${ }^{6}$, Monica Fletcher $^{7}$, Sarah Masefield ${ }^{8}$, \\ Pippa Powell ${ }^{8}$ and Jeanette Boyd ${ }^{8}$
}

Affiliations: 'LAM Italia Onlus, Udine, Italy. ${ }^{2}$ LAM Action, Nottingham, UK. ${ }^{3}$ Service de Pneumologie, Hopital L. Pradel, Claude Bernard University Lyon 1, Lyon, France. ${ }^{4}$ UO Pneumologia e Terapia Semi-Intensiva Respiratoria - Servizio di Fisiopatologia Respiratoria ed Emodinamica Polmonare, Osp. San Giuseppe MultiMedica, Milan, Italy. ${ }^{5}$ ILD Center of Excellence, St Antonius Hospital, Nieuwegein, The Netherlands. ${ }^{6}$ Servicio de Neumología, Hospital Universitario del Henares, Coslada, Madrid, Spain. ${ }^{7}$ Education for Health, Warwick, UK. ${ }^{8}$ European Lung Foundation, Sheffield, UK.

Correspondence: Jeanette Boyd, European Lung Foundation, 442 Glossop Road, Sheffield, S10 2PX, UK. E-mail: jeanette.boyddeuropeanlung.org

ABSTRACT Lymphangioleiomyomatosis (LAM) is a rare lung disease that almost exclusively affects women and develops in about one in 400000 adult females. The European Lung Foundation worked closely with one of the patient organisations within its network, the European LAM Federation, to raise awareness of LAM at the 2014 European Respiratory Society International Congress in Munich, Germany. In addition, an invitation-only workshop with 45 individuals from 13 countries was held to discuss the priorities for women in Europe living with the disease. The need for ongoing collaboration to improve knowledge of this rare lung condition with healthcare professionals across Europe was highlighted.

@ERSpublications

Patient organisations and @EuropeanLung have surveyed the priorities of people living with LAM http://ow.ly/10gTld

This article has supplementary material available from openres.ersjournals.com

Received: Dec 182015 | Accepted after revision: March 192016

Support statement: The work described in this article was supported by the European Lung Foundation. Funding information for this article has been deposited with FundRef.

Conflict of interest: S. Masefield, P. Powell and J. Boyd are employees of the European Lung Foundation. Further disclosures can be found alongside this article at openres.ersjournals.com

Copyright $\odot$ ERS 2016. This article is open access and distributed under the terms of the Creative Commons Attribution Non-Commercial Licence 4.0. 


\section{Introduction}

Lymphangioleiomyomatosis (LAM) is a rare lung disease that almost exclusively affects women and develops in about one in 400000 adult females [1]. As a systemic disease, it is associated with cystic destruction of the lung, pleural effusions and abdominal tumours, and arises sporadically [2]. It also affects between $30 \%$ and $40 \%$ of women with tuberous sclerosis complex (TSC) [2], a genetic disorder that causes tumours to form in many different organs, including the lungs [3]. The discovery of the close connection between these two diseases has led to a broader understanding of LAM [4]. There is currently no cure for LAM but increasing levels of research, including the first large-scale clinical trial for women with LAM, in which it was demonstrated that the use of the drug sirolimus could have beneficial effects, have led to progress in understanding the disease $[2,5]$.

\section{Background}

Due to its rarity, LAM can be an isolating illness and its impact varies considerably from patient to patient. Research into the impact that LAM has on the quality of life of patients and their caregivers is scarce. However, a study in 2014 directly aimed to capture perceptions from patients about the ways that LAM affects their lives. Both physical symptoms and psychological experiences of LAM were described, with the most commonly experienced shown in table 1 [6].

In response to the need for further information and support for people with LAM, and to fund research into the disease, several LAM organisations and groups have been established in the last two decades, beginning with the LAM Foundation in the USA in 1995. The European LAM Federation was founded in 2010, and is a European network for LAM patient and advocacy groups; it intends to raise the visibility of LAM among healthcare professionals and the public. Its aims, over the next few years, include the creation of a LAM registry in Europe, which will help to share knowledge of LAM and give patients the opportunity to advocate with one voice [7].

The European Lung Foundation (ELF) has had a working relationship with the European LAM Federation since 2011 when its founder became a member of the ELF Patient Advisory Committee. The organisation is also a member of the ELF patient organisation network, which brings together respiratory patient organisations in Europe to network, share learning and raise awareness of common issues.

Late in 2013, Iris Bassi, President of LAM Italia and the European LAM Federation, approached the ELF to discuss holding an event to raise awareness of LAM at the 2014 European Respiratory Society (ERS) International Congress in Munich, Germany. The ELF works with the ERS to ensure that the patient's voice is heard at Congress and to help healthcare professionals improve their understanding of the patients' perspective.

Together, the ELF and the European LAM Federation planned two events at the Congress: a priority-setting workshop, bringing together a wide range of stakeholders to discuss key issues in LAM treatment and research; and a LAM-focused symposium, where leading specialists could give presentations on LAM and the outcomes from the workshop could be presented. These Congress platforms provided beneficial opportunities for information exchange from across many countries [8].

\section{Priority-setting consultation activities}

Online survey

It was agreed by the European LAM Federation and ELF that a multilingual online survey to identify the most important priorities for people affected by the condition would be developed in order to formulate the areas to focus on in the workshop discussions.

\section{TABLE 1 Physical symptoms and psychological experiences of lymphangioleiomyomatosis}

Primary physical symptoms

Shortness of breath

Low energy (or fatigue)

Cough

Sensations in the chest

Common psychological experiences

Frustration

Worry

Loss of identity

Embarrassment or guilt

Healthy defiance against the disease

Two of the physical symptoms described were present for all participants in the study: shortness of breath and low energy. Although less commonly mentioned, cough was also present in many participants as well as a sensation of heaviness or aching in the chest. The psychological experiences listed are those that were most commonly described by participants. 
The survey was designed using an iterative process, with the involvement of both staff and patients from both organisations. It was based around a set of priority topics that the European LAM Federation identified through their experience of working with LAM groups within their member network and individual LAM patient experts in the field. The LAM groups within the network represented individuals from several European countries: Austria, France, Germany, Ireland, Italy, Spain, Sweden and the UK.

The survey covered five key areas: LAM diagnosis, treatment, care, living with LAM and research. The draft list of statements identified within each of these areas was circulated in English to national LAM organisations from the aforementioned countries, who made additions and amendments until consensus was reached on the statements to be included in the survey.

To avoid bias in statement selection and to ensure that the statements included were relevant to the target audience across Europe, any statements that were country-specific were excluded and an open field was included at the end of the survey to capture any additional priority statements or topics of importance to the respondents that may not have been identified through the survey design process. In total, there were 26 statements included in the survey.

The survey structure was designed so that respondents were invited to rate the importance of a number of statements within each of the five key areas. The survey was completed anonymously, although respondents were given the option to submit their e-mail address in order to receive future updates.

The survey did not require separate written consent to be provided by respondents as ethical considerations were met, which included providing adequate information at the start of the survey including: a clear statement of the purpose of the survey; who conducted and funded the survey; and an explanation of the purposes for which the data were to be used.

The survey was made available for a period of 2 months in seven European languages (English, German, Italian, French, Spanish, Swedish and Norwegian). The translations into these languages were undertaken by national member representatives of the European LAM Federation who were fluent in English and the survey was made available via the SurveyMonkey platform (www.surveymonkey.net).

A snowball sampling method was used to disseminate the survey with the aim of reaching and involving as many patients from as many countries as possible.

The existing ELF professional, patient and organisational networks were primarily used to disseminate the survey. The survey was subsequently shared with their contacts and/or members. In addition, the European LAM Federation used their member network to disseminate the survey and social media channels of both organisations were used. The challenge to this approach is that although specific groups of patients can be targeted in this way, there is no way of calculating the actual reach or response rate.

A total of 572 surveys were completed and these were made up of 87\% individuals diagnosed with LAM, $7 \%$ family members/caregivers, $4 \%$ healthcare professionals and $2 \%$ identified as "other". The survey respondents were distributed geographically from 39 countries across six continents as follows and as shown in figure 1: Europe (Italy, UK, France, Germany, Spain, Sweden, Switzerland, Austria, Ireland, Poland, Belgium, Denmark, Austria, Belgium, Croatia, Denmark, the Netherlands, Norway, Croatia, Russia, Luxemburg, Portugal and Romania); Africa (Egypt and South Africa); Asia (Israel, Turkey, Japan, Singapore and Pakistan); North America (the USA, Canada and Mexico); South America (Argentina, Chile, Brazil, Colombia and Peru); and Australia.

FIGURE 1 Geographical percentage distribution of survey respondents by continent.

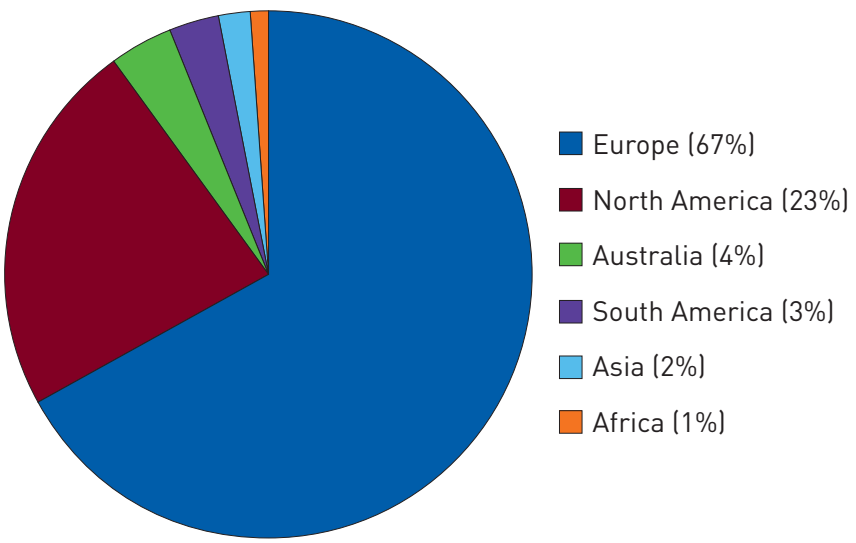


The statements for each of the five areas were analysed and ranked in priority order. A six-point rating scale was used for each statement, which included "top priority", "high priority", "important but not urgent", "not a priority but needs doing", "action not needed" and "not sure". The number of responses given for "top priority" and "high priority" for each area were combined and converted to percentages to identify the top 10 priorities across all areas, as shown in table 2.

Of the 26 priority statements, all but one was rated as a high priority by $>50 \%$ of respondents (supplementary table S1). Across the top 10 priorities, there were fewer respondents who rated the statements as low priority (between $4 \%$ and $10 \%$ of respondents) compared with the bottom five priority statements (statements $22-26$ in table S1), which received between $21 \%$ and $33 \%$ of respondents rating them as low priority. The lower priority rating was calculated by combining the number of rating responses given for "important but not urgent" and "not a priority but needs doing", and converted to percentages.

The open response answers were translated into English by a professional translation agency and then analysed thematically by the ELF. All comments were subsequently reviewed and verified by the European LAM Federation. The top 10 list was then used as the basis for discussion at the priority-setting workshop.

\section{Workshop and symposium}

The aim of the workshop was to bring together a range of stakeholders including those affected by LAM (patients and caregivers/family members) and people working in the field (healthcare professionals, researchers and industry representatives) to discuss the priorities identified by the online survey, and agree on any actions needed to improve LAM treatment and care now and in the future. In addition, the workshop aimed to identify the information needs of patients and their family members/caregivers, and whether new or additional European guidance was needed.

The workshop took place at the ERS International Congress in Munich in September 2014 with 45 participants from Austria, Croatia, France, Germany, Ireland, Italy, the Netherlands, Poland, Portugal, Spain, Sweden, the USA and the UK. Patient organisations represented at the workshop included LAM Italia (Italy), LAM Selbsthilfe Deutschland (Germany), LAM Academy (Sweden), Asociacion Espanola De Linfangioleiomiomatosis (Spain), LAM Action (UK), LAM Support Ireland, LAM France, LAM Group Bremen (Germany) and the European Tuberous Sclerosis Complex Association.

The findings from the workshop discussions are presented in the following Priorities section. Following the workshop, the outcomes from both the survey and workshop discussions were presented in an ERS symposium attended by delegates from across the world.

\section{Priorities}

A discussion of each of the 10 priorities and why they are of importance to patients follows. The priorities have been grouped for clarity of discussion into the themes: diagnosis (priorities 1, 5 and 9); drug availability, access and research (priorities 2, 6 and 8); treatment and lung transplant (priorities 4 and 10); and care (priorities 3 and 7).

\section{Diagnosis}

Priority 1: Improving methods of identifying patients with active disease

Priority 5: Improving noninvasive diagnosis

Priority 9: Improving diagnostic biomarkers

The importance of improving diagnosis of LAM was evident in the outcomes of the survey, where the top priority was to improve methods of identifying patients with the active disease, along with improving

\section{TABLE 2 Top 10 priorities from the lymphangioleiomyomatosis (LAM) survey}

1) Improving methods of identifying patients with active disease

Priorities are listed in descending order of importance. EU: European Union. 
noninvasive diagnosis (methods, accuracy and widespread use) and diagnostic biomarkers (or indicators) of the disease. Due to the rarity of LAM, there is limited awareness among healthcare professionals of the disease and its symptoms, which can lead to both misdiagnosis and a delay in diagnosis [9]. Now there is a recognised drug treatment for LAM that slows and sometimes stops progression of the disease; it is crucial that patients are diagnosed as quickly as possible so that optimal treatment can be planned.

Synergies can be seen with the field of idiopathic pulmonary fibrosis (IPF), another rare pulmonary disorder, which has recently seen the development of two potential treatments given individually or in combination $[10,11]$. Finding an effective treatment has led to the patient and professional community coming together to ensure that the new treatment is accessible; specifically, a patient-professional charter has been developed [12]. This patient-physician charter highlights the inequalities and unmet needs in IPF care across Europe, and aims to be a call to action for healthcare policymakers to drive improvement in European IPF care.

Until several years ago, many women with suspected LAM underwent invasive diagnostic procedures but this situation has started to change, in large part, due to the advent of biomarker research and identification of specific biomarkers for LAM that can be looked for in order to diagnose LAM noninvasively and with greater accuracy [13].

A study in 2012 evaluated serum biomarkers as diagnostic tools [14]. Serum vascular endothelial growth factor-D was one of the biomarkers examined. Applying ERS guideline criteria alongside this biomarker allowed a definite diagnosis without biopsy in 79\% of the study's cohort. Ensuring awareness of these biomarkers can reduce the need for surgical biopsy, and meet the patient priority of reducing invasive procedures. It was strongly stated by the patients that the ERS guidelines on LAM should be updated to include the latest diagnostic testing information as this is now out of date [1].

In November 2014, a LAM Biomarker Innovation Summit took place in Atlanta, GA, USA, bringing together a group of scientists with biomarker expertise to promote the case for how new biomarkers could help patients make better decisions about health and care [15].

The ELF has a role to play to ensure that patients can be informed about the role of biomarkers and the potential implications for their future treatment. The ELF is also working hard, together with the ERS and as part of several EU projects $[16,17]$, to highlight the importance of the impact of biomarkers on the potential of personalised medicine and the advantages that can bring for patients with lung conditions.

Drug availability, access and research

Priority 2: Availability of drugs for LAM in all European Union countries

Priority 6: Patients can access treatments such as low-dose sirolimus/everolimus

Priority 8: Opportunity for patients to participate in clinical trials

Priorities in this area for LAM patients focus on widening the availability of and access to drugs such as sirolimus in all European Union (EU) countries, as current access to them varies. Patients would also like more information about combination therapies and improved opportunities to participate in LAM clinical trials.

There is a wide range of drugs prescribed for the treatment of LAM and available across Europe. However, there is evidence to support the use of sirolimus due to a study in the USA that demonstrated beneficial effects in patients with LAM [2].

The MILES (Multicenter International LAM Efficacy of Sirolimus) clinical trial aimed to test the drug sirolimus (also known as rapamycin) to see whether treating LAM patients with sirolimus could improve their lung function. This placebo-controlled study ran for 12 months from December 2006 to September 2010, with 46 patients receiving sirolimus and 43 receiving placebo. The study demonstrated that sirolimus treatment could be useful in treating patients with moderate to severe LAM, as it stabilised lung function and improved quality of life. The study also recommended that treatment decisions should be made on an individual basis and that further clinical trials were needed to evaluate the long-term safety of sirolimus [2].

In addition to the MILES trial reported in 2011, and subsequent to the workshop, another paper [18] followed patients on sirolimus over a longer period of time, with 38 patients treated between 3.5 and 5 years, and in May 2015, the US Food and Drug Administration formally approved sirolimus to treat LAM.

Continued research into the preventative role of sirolimus was a priority for workshop participants. The ERS guidelines for the diagnosis and management of LAM, published in 2010, state that sirolimus should not be prescribed routinely outside clinical trials for pulmonary LAM [1]. However, acknowledging that there may be some possible treatment benefit, the guidelines suggest that sirolimus may be considered on an individual basis in patients with rapid decline in lung function or symptoms, after careful evaluation of risk/benefit ratio in a specialist/referral centre. Patients at the workshop felt that the ERS guidelines would benefit from being updated, especially in regard to sirolimus and other therapies, noting that in the period since the guidelines were published in 2010, there have been important developments. 
A more recent study took place from January 2010 to June 2012 with 24 patients from centres in the USA, France and Italy. This study assessed the efficacy and safety of everolimus in women with LAM and shows some initial evidence in the effectiveness of everolimus in improving pulmonary symptoms in patients with sporadic or TSC-associated LAM [19].

Patient registries and biobanks are a developing resource for research and are particularly important in rare diseases, in which accessing sufficient numbers of research cases can be challenging. In 1997, a registry to capture the demographic, clinical, physiological and radiographic features of people with LAM was established by the National Heart, Lung and Blood Institute of the National Institutes of Health in the USA, collecting the data of people with LAM at six clinical centres [20]. In the Netherlands, the biobank at the ILD Center of Excellence, St Antonius Hospital in Nieuwegein, is used for a nationwide registry of patients with LAM to enable countrywide insight, and enables participation in the global LAM registry of the LAM Foundation International Clinics programme to help identify patients and their availability for trials for research worldwide [21]. There is also a French national LAM registry (RE-LAM-CE) co-ordinated in Lyon.

The development of a European LAM registry to support research in larger clinical trials was a recommendation made at the workshop. There may be a role for the ERS to provide advice on tissue sharing. The increasing capture of LAM data internationally aims to increase awareness of the disease and its symptoms among both healthcare professionals and the wider public.

\section{Treatment and lung transplant}

Priority 4: Lung transplants should be available to more patients

Priority 10: The development of a European-wide organ donor service

Lung transplantation for LAM patients is now widely accepted as a therapeutic option in advanced pulmonary LAM [22] but as donated lungs are a scarce resource this option remains limited, as well as there being varying age limits for transplantation in different countries that means it is not always an option. In the 2005 Registry of the International Society for Heart and Lung Transplantation: Twenty-second Official Adult Lung and Heart-Lung Transplant Report [23], LAM accounted for $1.1 \%$ of all adult lung transplant recipients (January 1995 to June 2004). A 2005 review of patients in the USA who underwent primary lung transplantation for end-stage pulmonary LAM between January 1987 and December 2002 found that it is a valuable therapeutic option for patients and that it offers survival rates that are equivalent to or better than those of patients who received a lung transplant for other lung conditions [24]. A retrospective multicentre study of 44 patients in France who underwent lung transplantation for LAM between 1988 and 2006 was conducted, and found transplantation to be a satisfactory therapeutic option for end-stage respiratory failure in LAM [25].

However, the availability of lung transplants varies greatly between countries across Europe. LAM patients at the workshop expressly desired that lung transplants should be made more available to people with LAM and with shorter waiting times.

Eurotransplant is currently responsible for the allocation of donor organs in eight member states and introduced the Lung Allocation Score (LAS). The LAS aims to prioritise individuals on the waiting list by estimating medical urgency and probability of success after transplantation (www.eurotransplant.org). Expansion of such a European-wide organ donor service for patients could be beneficial. Although they recognise that these aims are ambitious and challenging, patients believe that increased collaboration across Europe would help improve access to treatment, including transplants, for all patients across the continent.

\section{Care}

Priority 3: All patients can access a specialist LAM centre

Priority 7: Improving care for patients in nonspecialist hospitals/services

A high priority for patients is the ability to access a specialist LAM centre for treatment and support. The LAM Foundation in the USA has developed or established links with a network of regional LAM clinics throughout the world with a view to improving the care and treatment of patients with LAM. This has included links with several established LAM centres in Europe (including in Barcelona, Spain; Dublin, Ireland; Lausanne, Switzerland; Lyon, France; Milan, Italy; Nieuwegein, the Netherlands; Nottingham, UK; and Zagreb, Croatia). The objective of the LAM Foundation Clinic Programme is to focus LAM care to medical institutions or hospitals that have the interest and expertise to deliver state-of-the-art, coordinated, multidisciplinary LAM care, and to perform co-operative research with other LAM clinics [26].

LAM specialists in Europe have also called for specialist LAM centres covering large geographical areas and close collaboration between them, whereby patients can be seen at least once a year by a doctor with expertise in LAM, and at a centre specialising in LAM diagnosis and treatment [13].

The EU is also currently looking to fund a European Rare Diseases network, which will aim to provide a network connecting healthcare providers and centres of expertise of highly specialised healthcare, for the 
purpose of improving access to diagnosis, treatment and the provision of high-quality healthcare for patients with conditions requiring a particular concentration of resources or expertise no matter where they are in Europe. If this network can be developed in the field of respiratory medicine, it provides a realistic and affordable possibility of developing a network of specialist LAM centres.

From the work of the ELF with patients with a variety of respiratory conditions, general views about multidisciplinary care suggest that it is difficult to specify in advance how this should be structured for any particular condition. What is of importance to patients is that they are consulted about the range of specialists they would like to be involved in their care, for psychological support to be offered, and that they have one key contact to co-ordinate access to information and services (this could be a specialist nurse, for example). The healthcare professionals involved in their care should also ensure that they liaise closely with each other to ensure a more holistic approach.

Workshop participants highlighted the potential benefit of training for specialists on LAM treatments and advances, as well as increasing their awareness of referral centres.

\section{Patient research priorities for LAM}

The importance of research for patients with LAM was also evident through the survey findings, which highlighted a desire for access to clinical trials and to be kept informed about the latest trials and results. As well as discussing the need for improved and increased access to clinical trials, the workshop participants also discussed their priorities for research topics. The topics agreed as important by all participants are listed in table 3.

There was no specific crossover between LAM priorities and those of another research priority exercise that the ELF ran with lung cancer patients. There were also no other research priorities found for LAM from other sources.

Several general issues with conducting research in this field were highlighted, including recognition that it is difficult to undertake a research study with sufficient numbers due to LAM being a rare disease, although it was felt that this is being addressed slowly through the introduction of national, European and international registries and biobanks, as discussed above. Another major challenge to research that was identified was the variance of treatment in different countries and that, in order for research and wider trials to be funded, greater collaboration is needed with the pharmaceutical industry. The links that the LAM patient organisation community have already forged with the medical and scientific community now need to be developed with the pharmaceutical industry.

As the understanding of the cell biology of LAM develops, it is becoming apparent that research in other disease areas could have implications for LAM. The translation of this research into the context of LAM would be welcomed by people living with LAM. One way of achieving this would be when applying for funding, i.e. the workshop participants suggested that researchers could link with other rare disease groups, and foster greater co-operation between US and EU research teams on topics such as cancer and epigenetics linked to LAM.

All stakeholders in LAM need to work together, to ensure that research can be adequately funded and coordinated. There are lessons to be learned from other respiratory diseases. Patient-powered research networks, such as the Patient-Centered Outcomes Research Institute (PCORI) in the USA [27], can be a way to ensure that patient data is collected and ready to be used for research across the globe. Examples in the respiratory field include chronic obstructive pulmonary disease. The aim of organisations such as the

\section{TABLE 3 Patient research priority areas}

\section{Diagnosis}

How to develop better, noninvasive methods for the early diagnosis of LAM

\section{Progression}

How to develop better information about the likely progression of LAM, perhaps through biomarkers How does oestrogen affect LAM?

An analysis of complications associated with the condition such as gynaecological complications Do lymphatics play a role in improved survival?

Drug treatment

How effective is sirolimus as a preventative drug?

An analysis of the ovarian toxicity of drugs

Why do some individuals develop resistance or do not respond to treatment?

LAM: lymphangioleiomyomatosis. 
PCORI is to ensure that people make informed healthcare decisions, and improve healthcare delivery and outcomes, by producing and promoting high-integrity, evidence-based information that comes from research guided by patients, caregivers and the broader healthcare community.

\section{Conclusions and recommendations}

This article aims to summarise an important piece of work in which LAM patient organisations, facilitated by the ELF, were able to discuss priorities in their diagnosis, treatment and care. This was strengthened by the attendance at the workshop of healthcare professionals to ensure that realistic and achievable priorities were identified, which also gives further external validity to the conclusions.

There is a number of methodological challenges to gathering patient perspectives and our approach is one of pragmatism as the primary aim was to involve as many patients as possible. By seeking to engage patients and gather views widely from across Europe, through surveys and workshops such as these, we are able to provide a valuable explorative approach and can highlight areas for future more rigorous studies.

Some of the specific challenges faced include the self-selection bias of the approach used, as individuals who complete a survey tend to have had a very good or very bad experience and are keen to communicate this, which may result in fewer responses from the moderate middle ground and bias the findings. In addition, computer literacy and internet access also limit those who are able to participate. However, involving patient organisations in consensus statements or task forces has become indispensable, especially for rare and orphan diseases [12], and patients' initiatives are essential to inform future clinical research, reveal structural problems in standards of care, gain political attention and ensure that goals are reflective of patients' needs, leading to improved outcomes; this has been headed up by activities in the mild to moderate hearing loss and mesothelioma research fields with organisations such as the James Lind Alliance [28, 29].

The priority-setting workshop provided valuable insight into those areas deemed to be of most importance to people with experience of LAM, and highlighted major concerns as well as identifying ways of improving diagnosis, treatment and care for all people with LAM. A range of information needs was also identified and in response to these, the ELF established a patient priorities project to develop a European website to link to information, support and resources about LAM throughout Europe, to be launched in 2016.

The pace and progress of research into LAM to date is encouraging, especially given its rarity. This brings hope to those affected and these individuals want to use this impetus to increase awareness of the condition, particularly with healthcare professionals. The call for more European-wide collaboration and co-ordination to raise awareness of this condition is central to achieving the changes that patients desire.

\section{References}

1 Johnson SR, Cordier JF, Lazor R, et al. European Respiratory Society guidelines for the diagnosis and management of lymphangioleiomyomatosis. Eur Respir J 2010; 35: 14-26.

2 McCormack FX, Inoue Y, Moss J, et al. Efficacy and safety of sirolimus in lymphangioleiomyomatosis. $N$ Engl $J$ Med 2011; 364: 1595-1606.

3 Curatolo P, Bombardieri R. Tuberous sclerosis. Handbook Clin Neurol 2007; 87: 129-151.

4 Taille C, Borie R, Crestani B. Current management of lymphangioleiomyomatosis. Curr Opin Pulm Med 2011; 17: 374-378.

5 The LAM Foundation. Therapeutic Strategies for LAM. www.thelamfoundation.org/medical-providers/lam-therapy Date last accessed: November, 2015.

6 Belkin A, et al. "Getting stuck with LAM": patients' perspectives on living with lymphangioleiomyomatosis Health Qual Life Outcomes 2014; 12: 79.

7 Bassi I. Patient organisation focus: European LAM Federation. www.europeanlung.org/en/news-and-events/news/ patient-organisation-focus-european-lam-federation Date last updated: June 18, 2014.

8 European Lung Foundation. Gill Hollis: LAM at the European Respiratory Society. www.europeanlung.org/en/ projects-and-research/projects/european-patient-ambassador-programme-(epap)/being-a-patient-ambassadors/ blogs/ Date last accessed: November, 2015.

9 Fletcher M, Bassi I. Patient voices at the ERS International Congress 2014: highlighting LAM. Breathe 2014; 10: 283-285.

10 Noble PW, Albera C, Bradford WZ, et al. Pirfenidone in patients with idiopathic pulmonary fibrosis (CAPACITY): two randomised trials. Lancet 2011; 377: 1760-1769.

11 Richeldi L, du Bois RM, Raghu G, et al. Efficacy and safety of nintedanib in idiopathic pulmonary fibrosis. $N$ Engl J Med 2014; 370: 2071-2082.

12 Bonella F, Wijsenbeek M, Molina-Molina M, et al. European IPF Patient Charter: unmet needs and a call to action for healthcare policymakers. Eur Respir J 2016; 47: 597-606.

13 Bassi I, Harari S. LAM: lymphangioleiomyomatosis the patient and healthcare professional perspective. Breathe 2014; 10: 331-332.

14 Chang WY, et al. Clinical utility of diagnostic guidelines and putative biomarkers in lymphangioleiomyomatosis. Respir Res 2012; 13: 34.

15 The New Zealand LAM Charitable Trust. News: December 2014 - LAM Biomarkers Innovation Summit. www.lam.org.nz/News.html Date last updated: December, 2014. Date last accessed: November, 2015.

16 Shaw DE, Sousa AR, Fowler SJ, et al. Clinical and inflammatory characteristics of the European U-BIOPRED adult severe asthma cohort. Eur Respir J 2015; 46: 1308-1321. 
17 European Asthma Research and Innovation Partnership (EARIP). http://earip.eu/ Date last accessed: February 2016.

18 Yao J. Sustained effects of sirolimus on lung function and cystic lung lesions in lymphangioleiomyomatosis. Am J Respir Crit Care Med 2014; 190: 1273-1282.

19 Goldberg HJ, et al. Everolimus for the treatment of lymphangioleiomyomatosis: a phase II study. Eur Respir J 2015; 46: 783-794

20 Maurer JR, et al. Lung transplantation in the management of patients with lymphangioleiomyomatosis: baseline data from the NHLBI LAM Registry. J Heart Lung Transplant 2007; 26: 1293-1299.

21 Struik MHL, et al. The importance of biobank and nationwide registry for lymphangioleiomyomatosis in a small sized country. Expert Opin Orphan Drugs 2015; 3: 393-401.

22 Benden C, et al. Lung transplantation for lymphangioleiomyomatosis: the European Experience. J Heart Lung Transplant 2009; 28: 1-7.

23 Trulock EP, et al. Registry of the International Society for Heart and Lung Transplantation: twenty-second official adult lung and heart-lung transplant report 2005. J Heart Lung Transplant 2005; 24: 956-967.

24 Kpodonu J, et al. The US experience with lung transplantation for pulmonary lymphangioleiomyomatosis. J Heart Lung Transplant 2005; 24: 1247-1253.

25 Reynaud-Gaubert $\mathrm{M}$, et al. Lung transplantation for lymphangioleiomyomatosis: the French experience. Transplantation 2008; 86: 515-520.

26 The LAM Foundation. International LAM Clinics. www.thelamfoundation.org/patients/international-lam-clinics Date last accessed: November 2015.

27 Daugherty SE, et al. Patient-powered research networks: building capacity for conducting patient-centred clinical outcomes research. J Am Med Inform Assoc 2014; 21: 583-586.

28 Henshaw H, Sharkey L, Crowe D. Research priorities for mild-to-moderate hearing loss in adults. The Lancet 2015; 386: 2140-2141.

29 Stephens RJ, Whiting C, Cowan K. Research priorities in mesothelioma: a James Lind Alliance Priority Setting Partnership. Lung Cancer 2015; 89: 175-180. 